\title{
Designing of an Indoor Visible Light Communication Transceiver for Data Transmission using White LED
}

\author{
Ha Duyen Trung, Do Trong Tuan \\ Department of Aerospace Electronics, School of Electronics and Telecommunications \\ Hanoi University of Science and Technology \\ Emails: \{tuan.dotrong, trung.haduyen\}@hust.edu.vn
}

\begin{abstract}
In this paper, a prototype of full-duplex indoor Visible Light Communication (VLC) transceiver is designed to demonstrate an optical wireless system based on Universal Serial Bus (USB) port for data transmissions using white Light Emitting Diode (LED). Based on VLC technology, the designed system consists of transmitters and receivers can achieve data rate of $161.2 \mathrm{Kbps}$ error free at the distance of $92 \mathrm{~cm}$ between two computers for real-time text and image transmission over indoor environment. Experiment results show that for text transmission, symbol error rate (SER) does not vary data rate at some transmission distances, whereas for image transmission, minimum square error (MSE) and peak signal to noise ratio (PSNR) indicate that the received image and transmitted image were almost similar.
\end{abstract}

Keywords: Visible Light Communication (VLC), LED, Transceiver design.

\section{INTRODUCTION}

White Light Emitting Diodes (LEDs) has recently been promising as a new growth technology which is expected to replace traditional illumination sources. White LEDs has more advantages compared to the existing incandescent in terms of long life expectancy, high tolerance to humidity, low power consumption, etc. Their applications include numeric displays, flash-lights, vehicle brake lights, traffic signals and the ubiquitous power-on indicator light [1-4].

Besides the above mentioned unique advantages of the white LED as an illumination light source, indoor communication systems using white LEDs are increasingly growing because there are many devices using lightings in our offices, home, home appliances including TVs and so on. The typical LED has special characteristics to light on and off very fast at ultra-high speed. By using visible light for the data transmission, problems related to radio communications are almost resolved such as transmitting at ultra-high speed, harmless for human body [5-6]. The LED based visible light communication is interpreted as a convergence communication technology which is not only used as a lighting device, but also to be used as communication device [7]. It is a kind of indoor optical wireless communication (OWC) that uses 'visible light' ray as communication medium.

To date, there are a number of research projects in VLC have been carried out [8-11]. In [8], the VLC Consortium (VLCC) established in 2003 with major companies in Japan aiming to publicize and standardize the visible light communication technology, which has been discussed and evaluated in the various industry fields. This ubiquitous and human interface technology provides the communication capability through LED lightings in our offices and homes, LED commercial displays, LED traffic signals, LED small lamps on electronic home appliances, etc in our daily life. Then, the European OMEGA project [9], the Wireless World Research Forum (WWRF) [10] and a forthcoming IEEE standard for VLC [11] have dedicated to this research area. In addition, research works on high speed data transmission, channel characteristics and modulation schemes have been proposed for the possibility of designing a new model that could fit the present infrastructure for indoor applications [1216]. In [12] Vucic et. al. reported a wireless visiblelight link operating for the first time at $125 \mathrm{Mb} / \mathrm{s}$ 
over $5 \mathrm{~m}$ distance indoor by use of on-off-keying (OOK) at the bit-error-ratios (BER) below 2.10-3. Hoa et. al. described in [13] a high-speed VLC link of $10 \mathrm{Mb} / \mathrm{s}$ data rate using a white-light LED and OOK nonreturn-to-zero (NRZ) modulation. In [14] authors reviewed the recent improvements on bandwidth extension and methods increa-sing transmission speed and discussed major application areas of the VLC. In [15] Mesleh et. al. proposed a power and bandwidth efficient pulsed modulation technique for OWC. Moreover, in [16] a data rate of $20 \mathrm{Mb} / \mathrm{s}$ achieved in a $1 \mathrm{~m}$ free space transmission by using the quaternary amplitude shift keying (4-ASK) modulation with digital filtering to enhance the direct modulation speed of white LED in VLC system.

In the most recently, there has been increasing research interest in VLC transceiver system design and demonstration depending on the indoor application scenarios [17-2]. In [17] and [18], authors investigated wireless optical transceiver especially focusing on the high speed and short range visible communication with expectation to be used as a peripheral interface of hand-held devices such as mobile phones, notebook computers, digital cameras. In [19] Png et. al. presented VLC audio transmitter and receiver circuit that can be integrated with LED reading lights above passengers' seats for the development of wireless airline entertainment systems. Png also described in [20] a circuit construction of VLC mass-storage prototypes that enable file transfers between the SD (Secure Digital) memory card and the PC via white-LED light. The entire system consists of a pair of identical whiteLED transceivers: one is connected to the PC, whereas the other is connected to the SD-card subcircuit. File transfer operates at 19200 bps. In [21] Rajbhandari et. al. presented divide constraints and design considerations of high-speed integrated VLC system to demonstrate a preliminary result of a MIMO system implementation operating at a data rate of $1 \mathrm{~Gb} / \mathrm{s}$. In addition, VLC transceiver designs were implemented on a field programmable gate array (FPGA) [22-24], in which the digital basebands for the transmitter and the receiver are implemented using two separate FPGAs. Thus far, the reported works mainly focused on basic functionality demonstration or signal processing algorithms and techniques to improve the data rate. However, the detail fundamental design consideration of VLC transceiver including system model, transmitter and receiver schematic, transceiver layout and circuit modules, testing scenarios of indoor full-duplex transceiver, text and image demonstration and especially, the threshold-cut value for Photodiode (PD), have not been clarified.

In this paper, we therefore report a design approach of a full-duplex transmission system using white LED based on USB port with 2 Mbps data rate for indoor visible light application. To our knowledge, this is the first report of such a method. Self-written software is also developed to achieve the data transmission and real-time text/image transmissions between two PCs. Design and analysis of an integrated VLC receiver with an USB 2.0 interface for PC or Note Book has been introduced in [25] and [26]. USB interface is a universal standard of external bus to specify the connection and communication between PC and electronic devices. The data rate and transmission distance could be improved by increasing the number of LEDs at transmitter and PDs at the receiver.

The rest of the paper is organized as follows: section II introduces the system model and transceiver design including system model, transceiver design and transceiver testing system setup. Section III gives system experimental results. Finally, the conclusions and future work are given in Section IV.

\section{SYSTEM MODEL AND TRANSCEIVER DESIGN}

\section{A. System model and transceiver design}

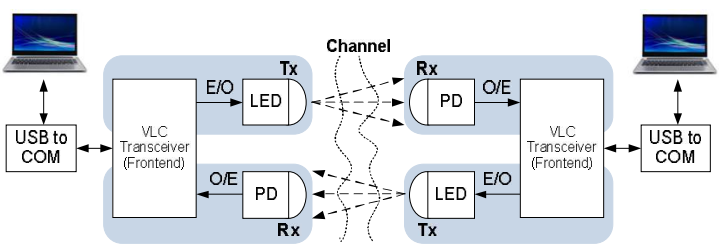

Fig. 1. A systematic block diagram of the fullduplex indoor VLC transceiver using white LEDs

A systematic block diagram of the full-duplex indoor VLC transceiver using white LEDs is illustrated in the Fig. 1. From the left side, at the transmitter, input information signals (text, image) generated from the first PC transmit to VLC 
transceiver via the USB to COM module. This module uses PL2303 (serial number MH000745) to connect USB port of the computer for signal conversion from differential values to TTL 3.3 peakto-peak voltage value. The VLC transceiver plays an important role of buffer to amplify the voltage to an adequate level in order to drive LED lighting to the air channel. The white LED ( $5 \mathrm{~mm}$ Luxeon) with the transmitting power of $1 \mathrm{~W}$ and the beam width of $120^{\circ}$ is used as a converter from electrical source to optical (E/O) transmission source. OOK/NRZ signal for intensity modulation (IM) and direct detection (DD) schemes is employed for widely reception in our design due to its simplicity and low cost. The whole circuit of transmitter design is presented in Fig. 2.

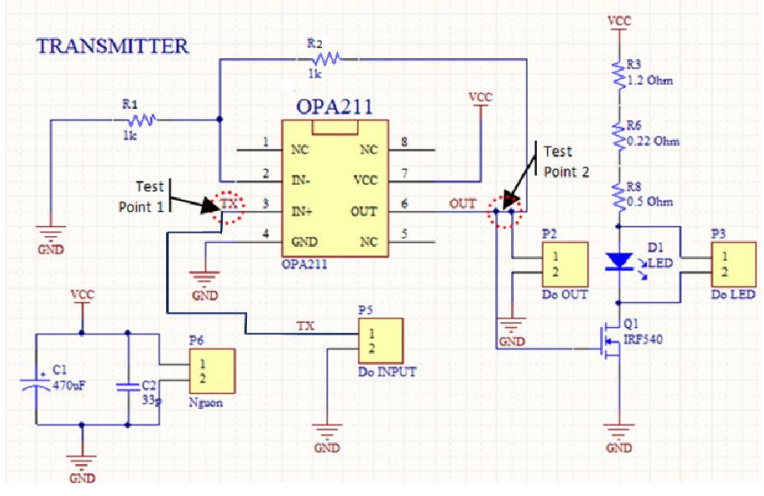

Fig. 2. The designed VLC transmitter schematic

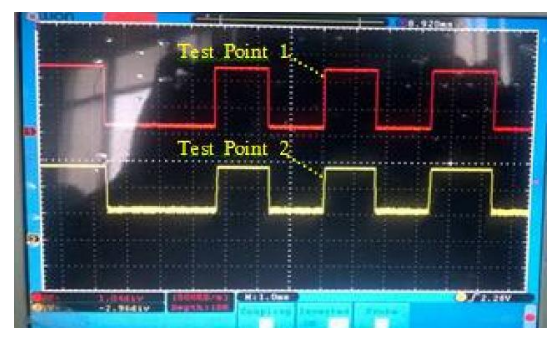

Fig. 3. Signals at Test Points when LED connected

In Fig. 2, an OPA211 module (Texas Instruments) has a high frequency response $(50 \mathrm{MHz})$, which is widely used to stabilize voltage for LED lighting. Generally, the output voltage of PL2303 is not high enough to trigger the MOS-FET IRF540. Therefore, OPA211 was used to amplify voltage supply for stimulating IRF540. The output signal of OPA211 can be expressed as

$$
V_{\text {out }}=V_{\text {in }}\left(1+\frac{R_{2}}{R_{1}}\right)=2 V_{\text {in }}
$$

In Eq. (1), $R_{1}=R_{2}=1 \mathrm{k} \Omega$, the input signal level TX at $\mathrm{IN}^{+}$port is from 0 to $3.3 \mathrm{~V}$. Then, the output signal level at OUT port achieves from 0 to $6.6 \mathrm{~V}$. However, with the $5 \mathrm{~V}$ input of OPA211 leads the output range from 0 to $5 \mathrm{~V}$ in case of no load or from 0 to $4.85 \mathrm{~V}$ when the LED is connected as the load through a MOS-FET IRF540. This value is used to control the IRF540 for supplying the white LED $3 \mathrm{~W}$ operating at $2.8-4.0 \mathrm{~V} / 350 \mathrm{~mA}$. It is noted from the figure 2 that $\mathrm{C} 1-\mathrm{C} 4$ capacitors are used to stabilize voltage and interference cancellation. The voltage levels measured at Test Point 1 and Test Point 2 when LED is connected to the output of op amps are illustrated in Fig. 3.

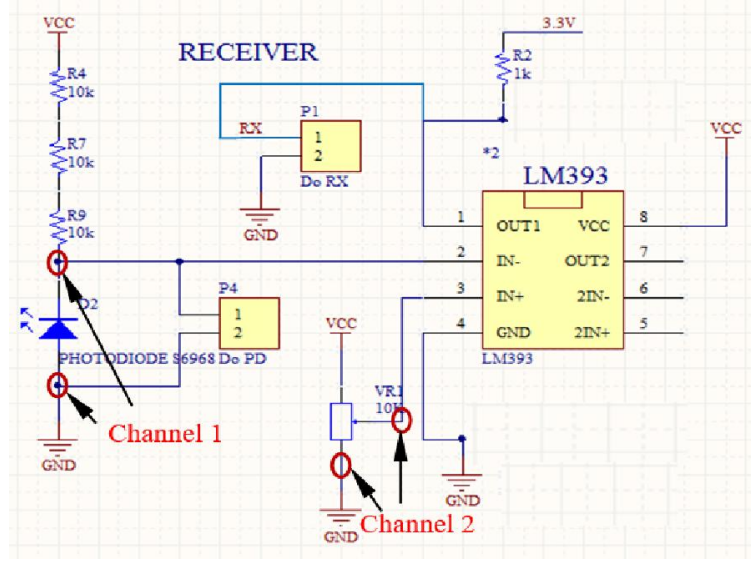

Fig. 4. The designed $V L C$ receiver schematic

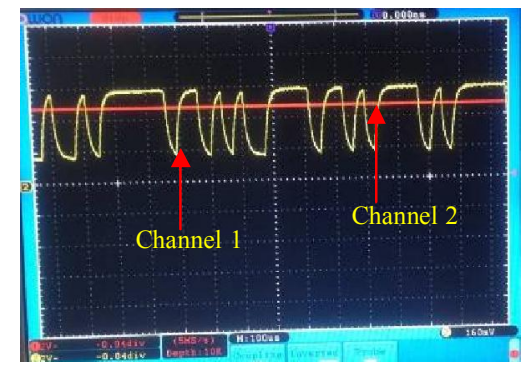

Fig. 5. The output signals at channel 1 and channel 2

At the receiver side, the PD has been adopted in optical communication systems with relatively large received optical power. The advantages of the PD 
include its low price and possible high reception bandwidth. The band-width of the photodiode is usually inversely proportional to its active receiving area because of the internal capacitance along with the receiving area. The whole circuit of VLC receiver schematic is presented in Fig. 4, where the receiver employs a commercially available Si PIN (Hamamatsu S6968) photodiode for optical to electrical $(\mathrm{O} / \mathrm{E})$ conversion with $150 \mathrm{~mm}^{2}$ effective active area, $14 \mathrm{~mm}$ active area size and $35^{\circ}$ half angle. The received optical signal is sent to a low pass filter followed by a custom amplifier for recovery. A decision module (LM393) is used as a single threshold voltage comparator to achieve the signal decision with a reference voltage level to obtain the original signal. The signal decision is called the threshold-cut value as illustrated in Fig. 5. This show case image is captured from the displayed monitor of an OWON SDS5032E oscilloscope. It is used to check real physical signal levels and to specify a right value of the threshold-cut level by experimental approach. The TTL signal is restored at the receiver by comparing received signal from PD's output (channel 1) with a threshold level obtained from a variable resistor's output (channel 2). The testing system including diagram and system setup is illustrated in Fig. 7 of the next subsection. The TTL signal after decision is sent to transceiver to convert from TTL signal to USB serial signal. It is noted that the transceiver module is composed of a transmitter and a receiver to realize the full-duplex optical wireless transmission. The full-duplex VLC transceiver layout and circuit modules are presented in Fig. 6 (a) and Fig. 6 (b), respectively.

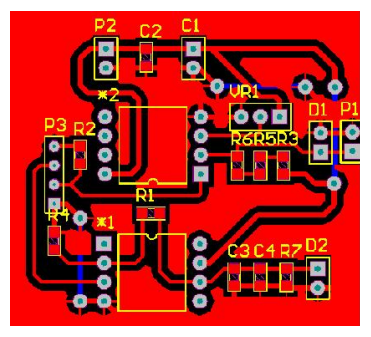

(a)

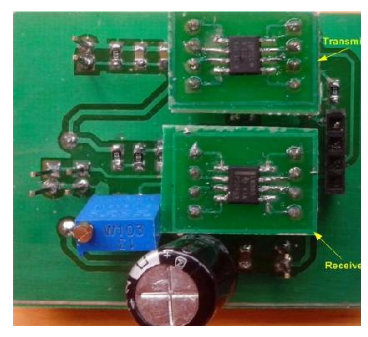

(b)
Fig. 6. Transceiver layout and circuit modules

(a) The VLC layout module, (b) The transceiver circuit

\section{B. Transceiver characteristic}

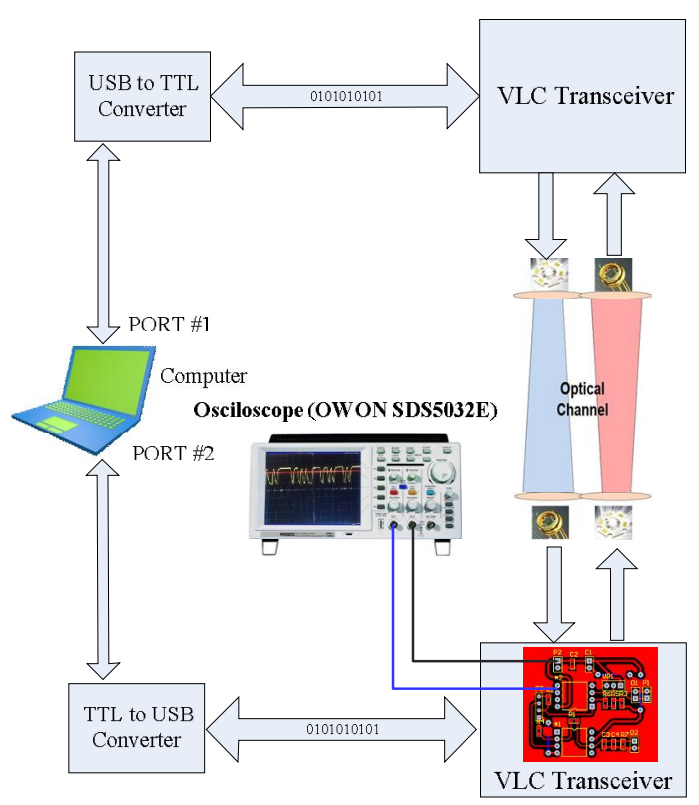

(a) Diagram of indoor VLC data transmission
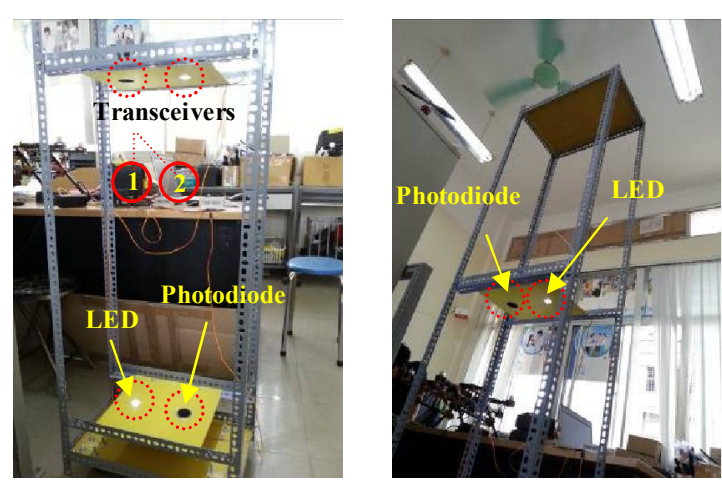

(b) Testing system setup

Fig. 7. The testing system of indoor full-duplex $V L C$

In the designed transceiver, power supply is provided by 3.3 VDC from USB port. The driver PL2303 is installed on Computers then connected to transceiver modules. The self-written software is used instead of the Hyper Terminal communication tool to transfer data (text) and image from Computers to the transceiver module via the selected USB port that can be configured by Baud Rate. Laptops treat the transceiver module as a visual serial port. The software application is written by using Visual C\#. The program is created to achieve 
real-time image transmission between two transceiver-settled computers. The image input is sent to the selected USB port and transmitted by the trans-ceiver module. After transmission through indoor visible light of sight channel, the opposite transceiver module interfaced on the second Computer, which received the bit streams and displayed them in real-time. Data transmission between two computers in full-duplex manners is shown in Fig. 7.

\section{SYSTEM DEMOSTRATION AND RESULTS}

As the full-duplex VLC transceiver design discussed in the Section II, in this section, we present two system demonstrations scenarios for text and image transmission, respectively. Fig. 7 illustrates the test-bed for indoor VLC transceiver demonstrations. The full-duplex communication links consist of forward channels and reverse channels. In the forward channel, LED (Tx) of first transceiver was attached on upper plane of the testbed shell and the photodiode (Rx) of the second transceiver was located at the lower plane of the shell. In the forward channel, photodiode (Rx) of first transceiver was attached on upper plane and the LED (Tx) of the second transceiver was located at the lower plane of the test-bed shell. The distance from the first transceiver and the second transceiver varies from $46.5 \mathrm{~cm}$ to $107.5 \mathrm{~cm}$ for different bit rates. Main parameters of LEDs and PDs used in the test-bed are shown in Table 1 and Table 2, respectively.

Table 1. Specifications of LED Luxeon [27]

\begin{tabular}{|l|c|c|}
\hline Parameters & Value & Unit \\
\hline Power & 3 & $\mathrm{~W}$ \\
\hline Forward Voltage & $2.8-4$ & $\mathrm{~V}$ \\
\hline Standard Current & 350 & $\mathrm{~mA}$ \\
\hline Luminous Flux & 45 & $\mathrm{Lm}$ \\
\hline Total include Angle & 160 & Degree \\
\hline Viewing Angle & 70 & Degree \\
\hline
\end{tabular}

Table 2. Specifications of Photodiode S6968 [28]

\begin{tabular}{|l|c|c|}
\hline Parameters & Value & Unit \\
\hline High-Speed Response & 50 & $\mathrm{MHz}$ \\
\hline Sensitivity & 0.63 & $\mathrm{~A} / \mathrm{W}$ \\
\hline Directivity & 35 & degree \\
\hline Lens Diameter & 14 & $\mathrm{~nm}$ \\
\hline Active Area & 150 & $\mathrm{~mm} 2$ \\
\hline Wavelengths & $320-1060$ & $\mathrm{~nm}$ \\
\hline
\end{tabular}

In the demonstrations, we used two PCs for data transmission each other, PCs connected to VLC transceivers via USB ports. Software for text and image transmission was design (Fig. 8) and installed at each PC. We firstly present text transmission of "Visible Light Communications" which includes 29 characters including spaces. These characters were transmitted at different data rates and transmission distances in order to estimate symbol error rate (SER). SER were calculated by average of 15 transmission times for each specific data rate and transmission distance.

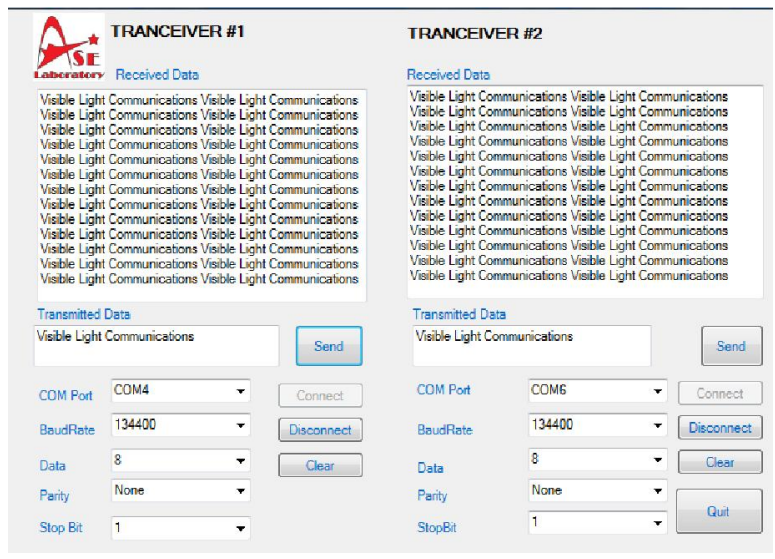

Fig. 8. GUI software for text and image transmission

Fig. 9 shows the SER for forward channel and reverse channel at different transmission bit rate. The transmission distances were varied from 46.5 $\mathrm{cm}$ to $107.5 \mathrm{~cm}$ for different bit rates of error free (Fig. 10). It can be observed that at the longest transmission distance of $107.5 \mathrm{~cm}$, bit rate of 57.6 kbps for forward channel and $38.4 \mathrm{kbps}$ for reverse channel without any symbol error were achieved. 
The highest bit rate of $161.2 \mathrm{kbps}$ and $134 \mathrm{kbps}$ was archived at transmission of $92 \mathrm{~cm}$ for forward channel and $38.4 \mathrm{kbps}$ for reverse channel when SER equal to zero.

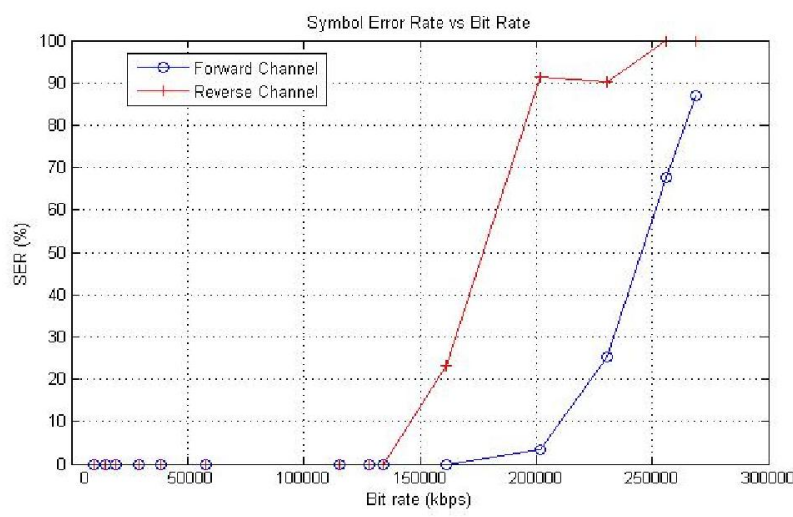

Fig. 9. Symbol error rate versus bit rate

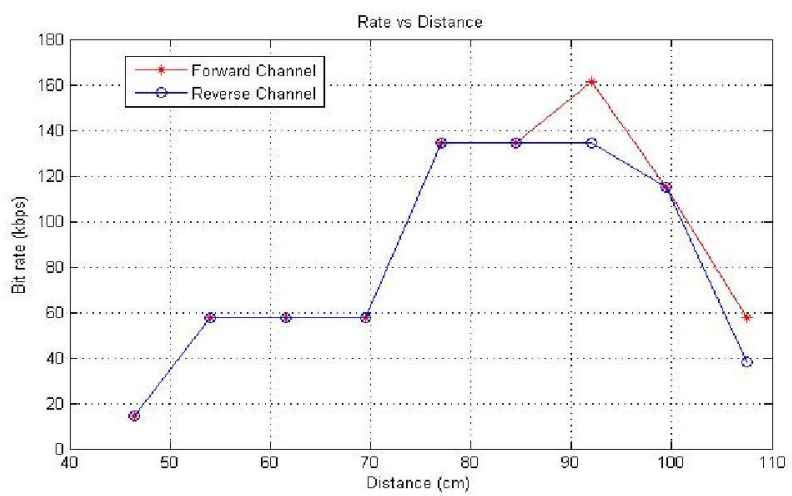

Fig. 10. Bit rate versus transmission distance

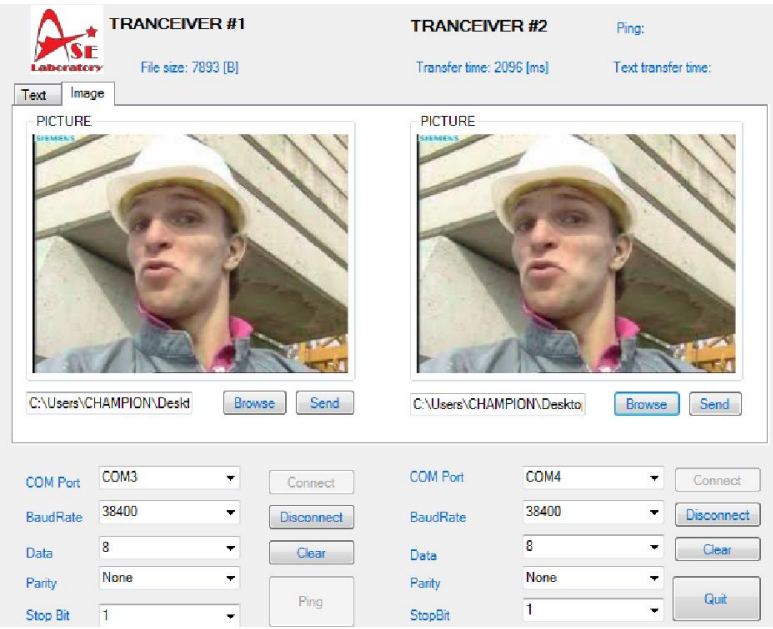

Fig. 11. Transmitted image (left side) and received image (right side)
In the second system demonstration with the purpose of the possibility of image transmission using the designed system, we used our software to send image files via COM port of Laptops to VLC transceiver. The image file "foreman" of 7.7 kBytes $(248 \times 203$ pixels $)$ and "Lena" of 31.6 kBytes $(200 \times 200$ pixels) were used for transmission at different transmission rates between $9.6 \mathrm{kbps}$ and 115.2 kbps. Fig. 11 presents transmitted image and received image.

Minimum Square Error (MSE) and Peak Signal to Noise Ratio (PSNR) were calculated based on transmitted and received images to analyze quality of the received image over the average of three transmission times. MSE and PSNR are respectively given as [29]

$$
\begin{aligned}
& \operatorname{MSE}=\frac{1}{N M} \sum_{i=1}^{N} \sum_{j=1}^{M}\left(y_{i, j}-\hat{y}_{i, j}\right)^{2} \\
& \operatorname{PSNR}_{(\mathrm{dB})}=10 \log _{10} \frac{\left(2^{b}-1\right)^{2}}{\mathrm{MSE}},
\end{aligned}
$$

where $M \times N$ is the image size in pixels, $y_{i, j}$ and $\hat{y}_{i, j}$ is the pixel value at the $(i, j)$ position of the transmitting and receiving image, and $b$ is the number of coded bits for image pixels.

Table 3 presents the received image at the receiver had good quality with MSE and PSNR equal to zero and infinity (Inf.). However, some images received without start byte or stop byte therefore they could not display correctly. The experimental results shown that data rate and transmission distance were limited at $9.6 \mathrm{kbps}$.

Table 3. Image transmission at distance of 99.5

\begin{tabular}{|c|c|c|c|c|c|c|c|}
\hline $\begin{array}{c}\text { Bit rate } \\
\text { (kbps) }\end{array}$ & 9.6 & $\begin{array}{l}14 . \\
4\end{array}$ & $\begin{array}{l}19 . \\
2\end{array}$ & $\begin{array}{l}28 . \\
8\end{array}$ & $\begin{array}{l}38 . \\
4\end{array}$ & $\begin{array}{l}57 . \\
6\end{array}$ & $\begin{array}{l}115 . \\
2\end{array}$ \\
\hline \multirow{2}{*}{$\begin{array}{l}\text { Delay } \\
(\mathrm{ms})\end{array}$} & $\begin{array}{l}9.4 \\
8\end{array}$ & $\begin{array}{l}6.7 \\
0\end{array}$ & $\begin{array}{l}5.0 \\
6\end{array}$ & $\begin{array}{l}3.3 \\
8\end{array}$ & $\begin{array}{l}2.7 \\
1\end{array}$ & $\begin{array}{l}2.2 \\
4\end{array}$ & 1.3 \\
\hline & $\begin{array}{l}37 . \\
1\end{array}$ & $\begin{array}{l}25 . \\
0\end{array}$ & $\begin{array}{l}18 . \\
6\end{array}$ & $\begin{array}{l}11 . \\
9\end{array}$ & 9.5 & $\begin{array}{l}6.5 \\
5 \\
\end{array}$ & 3.5 \\
\hline MSE & 0 & 0 & 0 & 0 & 0 & 0 & 0 \\
\hline PSRN & Inf. & Inf. & Inf. & Inf. & Inf. & Inf. & Inf. \\
\hline $\begin{array}{l}\text { Good } \\
\text { Quality }\end{array}$ & Yes & Yes & Yes & Yes & Yes & Yes & Yes \\
\hline
\end{tabular}
cm 
In order to investigate the capability for real-time data transmission over full-duplex VLC channels, the transmis-sion delay demonstrations were perform by using one Computer with two USB ports. This testing topology was used to eliminate the time different clock offset between transmitting and receiving Laptop. Fig. 12 shows the delay results for "Ping" packet containing just one character of ' $p$ '. The delay was calculated in unit of milliseconds by subtraction the receiving time to transmitting time. Delay of transmission for two investigated images also measured and shown in Fig. 13. The obtained transmission delays show the outperform of the designed VLC test-bed in term of real-time transmission capability with the value of $9.48 \mathrm{~ms}$ for "foreman" and $37.1 \mathrm{~ms}$ for "Lena" image at bit rate of $9.6 \mathrm{kbps}$ and $1.3 \mathrm{~ms}$ and $3.5 \mathrm{~ms}$ respectively at the transmission bit rate of 115.2 kbps (Table 3).

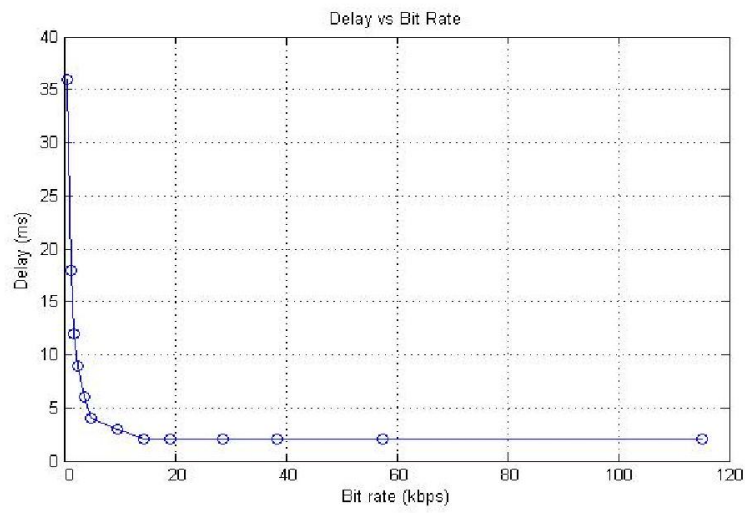

Fig. 12. Ping transmission delays versus bit rate

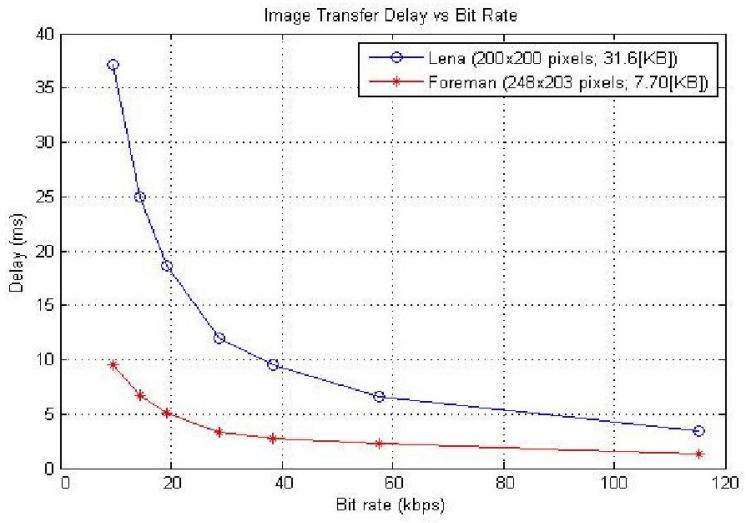

Fig. 13. Image transmision delays versus bit rate

\section{CONCLUSION AND FUTURE WORKS}

In this paper, practical transceiver designing indoor visible light communication systems, including transmitter and receiver devices was presented and discussed. Some experimental scenarios were demonstrated and analyzed in terms of transmission range, symbol error rate and transmission delay to confirm that data (text and images) transmission for indoor applications can be implemented by using white LEDs. In future works, we will improve effectiveness of designed VLC transceivers for real-time data transmission capability in various scenarios with considerations to light interferences from other light sources as well as effects of receiver concentrators.

\section{ACKNOWLEDMENT}

This research work is supported by the 2016 interdisciplinary project of Hanoi Univ. of Science and Technology between school of electronics and telecommunications and school of engineering physics.

\section{REFERENCES}

[1] Mukai, T., Nakamura, S.: White and WLEDs. OYO BUTURI 68(1999), 152-155.

[2] Tamura, T., Setomoto, T., Taguchi, T.: Fundamental characteristics of the illuminating light source using white light-emitting diodes based on InGaN semiconductors. IEEJ Trans. on Fundamentals and Materials, 120 (2000), 244-249.

[3] Taguchi, T.: Technological innovation of highbrightness light emitting diodes (LEDs) and a view of white LED lighting system. Optronics, 19(2000), 113-119.

[4] Nakamura, T.: Development of ZnSe-based white Light emitting diodes with longer lifetimes of over $10,000 \mathrm{hr}$. Electrical Engineering in Japan, 154(2006), 42-48.

[5] Komine, T., Nakagawa, M.: Fundamental analysis for visible light communication system using LED lights. IEEE Trans. on Consumer Elec., 50(2004), 100-107.

[6] Komine, T., Nakagawa, M., Integrated system of white LED visible-light communication and powerline communication. IEEE Trans. on Consumer Electronics 49 (2003) 71-79.

[7] Haruyama, S.: Visible light communication. IEICE Trans. on J86-A (2003) 1284-1291.

[8] VLCC, "Visible Light Communications Consortium," 2008. 
[9] "Home Gigabit Access project," funded by European Framework 7. Available at: http://www.ict-omega.eu/.

[10] "Wireless World Research Forum." Available at: http://www.wireless-world-research.org/.

[11] IEEE, "IEEE P802.15 Working Group for Wireless Personal Area Networks (WPANs)," 2008.

[12] J. Vucic, C. Kottke, S. Nerreter, K. Habel, A. Buttner, K. D. Langer and J. W. Waleski: $125 \mathrm{Mbit} / \mathrm{s}$ over $5 \mathrm{~m}$ Wireless Distance by Use of OOKModulated Phosphorescent White LEDs. Proc. of 35th European Conf. of Opt. Commun. (2009) 1-2.

[13] H. Le-Minh, D. O’Brien, G. Faulkner, L. Zeng, K. Lee, D. Jung, Y. Oh and E. T. Won.: 100-Mb/s NRZ Visible Light Communications Using a Postequalized White LED. IEEE Photonics Tech. Lett. 21 (2009) 1063-1065.

[14] Y. Zheng and M. Zhang: Visible Light Communications Recent Progresses and Future Outlooks. Proc. of Photonics and Optoelectronics Conf. (2011) 1-6.

[15] R. Mesleh, H. Elgala and H. Hass: Optical Spatial Modulation. Journal of Opt. Commun. and Netw. 3 (2011) 234-244.

[16] C. H. Yeh, Y. F. Liu, C. W. Chow, Y. Liu, P. Y. Huang and H. K. Tsang; Investigation of 4-ASK Modulation with Digital Filtering to Increase 20 Times of Direct Modulation Speed of White-Light LED Visible Light Communication System, Optics Express, 20 (2012) 16218-16223.

[17] A. Burton, C. Amiot, H. L. Minh and Z. Ghassemlooy: Design of an integrated Optical Receiver for Mobile Visible Light Communications. Proc. of PGNet, (2011)

[18] H. Shin, S.-B. Park, D.K. Jung, Y.M. Lee, S. Song, J. Park: VLC Transceiver Design for Short-Range Wireless Data Interfaces. Proc. of ICTC (2011) 689690.

[19] L. C. Png, S. X. Lim, A. R., B.-W. Chan, F. A. Hazman: Designs of VLC Transceiver Circuits for Reading Light Transmission of High-Quality Audio Signals on Commercial Airliners. Proc. ICCE (2014) 97-98.

[20] L. C. Png, N. L. Minh, L. Chen, K. S. Yeo: Designs of a Free-Space White-LED Mass-Storage Transceiver for SD-Card File Transfer. Proc. 3rd IEEE Workshop on Opt. Wire. Commun. (2012) 1260-1263.

[21] S. Rajbhandari, et. al.: High-Speed Integrated Visible Light Communication System: Device Constraints and Design Considerations. IEEE J. on Slec. Areas in Commun. 33 (2015) 1750- 1757.

[22] D. Terra, et. al.: Design, Development and Performance Analysis of DSSS-based Transceiver for VLC. Proc. EUROCON (2011) 1-4.

[23] F. Che, B. Hussain, L. Wu, C. P. Yue: Design and Implementation of IEEE 802.15.7 VLC PHY-I Transceiver. Proc. ICSICT2014 (2014) 1-4.
[24] B. Hussain, et. al.: Visible Light Communication System Design and Link Budget Analysis. J. of Ligh. Tech. 33 (2015) 5201- 5209.

[25] L. Ding, F. Liu, Y. He, H. Zhu, Y. Wang: Design of Wireless Optical Access System using LED. Optics and Photonics Journal, 3 (2013) 148-152

[26] I. E. Lee, et. al.: Design and Development of A Portable Visible-Light Communication Transceiver for Indoor Wireless Multimedia Broadcasting. Proc. ICED (2014) 20-24.

[27] Luxeon, Technical Datasheet DS25, Luxeon LED. Available at:

https:/www.sparkfun.com/datasheets/Components/ Luxeon-III.pdf; Last access: Apr. 10th 2015.

[28] Hamamatsu, Specifications of Photodiode S6968. Available at: https://www.hamamatsu.com/resources/pdf/ssd/s680 1_etc_kpin1046e.pdf; Last access: April 10th 2015.

[29] Z. Wang, H. R. Sheikh, and A. C. Bovik: Objective video quality assessment. The Handbook of Video Databases: Design and Applications (B. Furht and O. Marques, eds.), CRC Press, 2003.

\section{AUTHORS' BIOGRAPHIES}

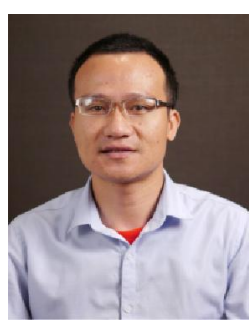

Ha Duyen Trung received the B.E. (2003), M.E. (2005), and D.E. (2009) degrees in Communications Engineering from Hanoi Univer-sity of Science and Technology (HUST) and Chulalongkorn University. $\mathrm{He}$ received Japanese International Cooperation Agency (JICA) scholarship awards for his MSc and PhD studies. In 2012, Dr. Trung spent 3 months as a visiting researcher at the University of Aizu, Japan. He has been a lecturer, Dept. of Aerospace Elec-tronics, School of Electronics and Telecommunication, HUST. His present research interests are in the area of optical wireless and MIMO communications.

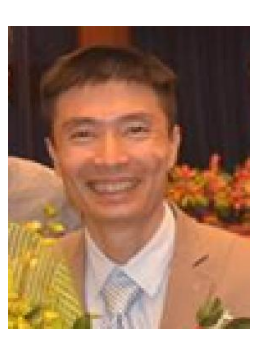

Do Trong Tuan received the B.E. (1997), M.E. (2000), and D.E. (2007) degrees in Communications Engineering from HUST. He is Associate Professor, Dept. of Aerospace Electronics, School of Electronics and Telecommunications, Hanoi University of Science and Technology. He was with Institute of Smart System Technologies, Klagenfurt Univ.-Austria and Chiba University- Japan, as a postdoctoral researcher during 2008-2009 and 2011-2012, respectively. His current research centers on Aeronautical and Space Communications and Navigations, Multimedia Mobile Embedded Systems and Optical Wireless Communica-tions. 